\section{Role of mesenchymal stem cell secretome as immunomodulator in periodontal diseases}

\author{
Desi S. Sari, ${ }^{1 *}$ Ernie Maduratna, ${ }^{2}$ Candra Bumi, ${ }^{3}$ I-Ketut Sudiana, ${ }^{4}$ \\ Fedik A. Rantam ${ }^{5,6}$
}

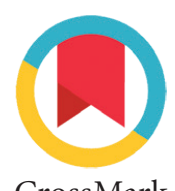

CrossMark

\title{
Abstract
}

Objective: The purpose of this review literature is to look at the mechanism of mesenchymal stem cell secretome in the regeneration of periodontal tissue.

Methods: This literature review was collected from several journals and research that has been done, where the application of Mesenchymal stem cells (MSCs) promises a favourable result for cellbased therapy in inflammatory diseases, immune system disorders, regeneration, and tissue repair.

Results: MSCs possess a potent immunomodulatory function, paracrine effects, and the ability to differentiate into different cells. Not only mesenchymal stem cells can replace damaged tissue or cells but also secrete secretome namely bioactive ingredients such as cytokines, chemokines, growth factors, pro-inflammatory, and anti-inflammatory which play an essential role in regulating the host immune system. Periodontal disease is an inflammatory disease that occurs in the teeth supporting structures. Lately, many therapies for periodontal disease utilized MSCs from gingival tissue and periodontal ligaments. Secretome activates endogenous MSCs to improve the immune system in periodontal disease that might create a better microenvironment of periodontal tissue. Conclusion: The secretome of MSCs has attracted a lot of attention and has the potential to repair and regenerate tissues including periodontal tissue.
'Department of Periodontics, Faculty of Dentistry, Universitas Jember, Jember, Indonesia ${ }^{2}$ Department of Periodontic, Faculty of Dental Medicine, Universitas Airlangga, Surabaya, Indonesia ${ }^{3}$ Department of Epidemiology and Biostatistics Population, Universitas Jember, Jember, Indonesia ${ }^{4}$ Department of Microscope Electron, Faculty of Medicine, Universitas Airlangga, Surabaya, Indonesia

${ }^{5}$ Department of Regenerative Medicine and Stem Cell Center, Universitas Airlangga/Dr Soetomo General Hospital, Surabaya, Indonesia

${ }^{6}$ Department of Stem Cells Research and Development Center, Universitas Airlangga, Surabaya, Indonesia

"Correspondence to: Desi S. Sari, Department of Periodontics, Faculty of Dentistry, Universitas Jember, Jember, Indonesia

desi_sari.fkg@unej.ac.id

Received: 24 September 2019

Revised: 22 March 2020

Accepted: 10 June 2020

Available Online: 1 December 2021

Keywords: Host response, Immunomodulator, Mesenchymal stem cells, Periodontal disease, Secretome

Cite this Article: Sari DS, Maduratna, E, Bumi C, Sudiana I, Rantam FA. 2021. Role of secretome mesenchymal stem cells as immunomodulator in periodontal diseases. Journal of Dentomaxillofacial Science 6(3): 139-146. D0I: 10.15562/jdmfs.v6i3.989

\section{Introduction}

Mesenchymal stem cells (MSCs) are unique, as these cells possessed multipotent, self-renewal, plasticity, and multilineage differentiation properties. MSC was first depicted in the 1970s who described cell populations derived from bone marrow that had fibroblast-like shapes and capable of producing adipocytes, chondrocytes and osteocytes. These cells grow in the form of colonies; therefore, they are called "colony forming units" or CFUs. ${ }^{1}$ Then, in 1990 these mesenchymal stem cells (MSCs) because they have multipotent properties. Differentiate into osteoblast cells, adipose cells and chondrocyte cells in culture media. Researches of MSCs began to develop in 2006. According to the International Society for Cellular Therapy (ISCT) there are at least three characteristics of the characteristics of MSCs. First, they form single-cell colony when cultured MSCs cells are attached to flask. Secondly, MSCs have to express CD105, CD73, CD90, and does not express CD45, CD34, CD14. CD79, CD 11a and CD-19 or MHC class II antigens. Lastly, MSCs can differentiate into osteoblasts, adipose and chondroblasts in vitro. ${ }^{2,3}$

Back in 2010, in vivo MSCs research toppled the new paradigm which considered MSCs as a replacement tissue, but has become a Medicinal signalling cell because of their function in vivo which they express the secretome by secreting soluble factor materials in the area of injury, and inflammation. ${ }^{4,5}$ Some evidence shows that MSC has relatively peculiar immunity. MSCs express relatively low histocompatibility antigen (MHC) and last longer when transplanted into allogeneic hosts than most of the other cell types. Also, MSC's capacity to detect changes in its microenvironment is mediated through several receptors for molecular signals. MSCs express various growth factors, pro-inflammatory and anti-inflammatory cytokines as well as chemokines and bioactive materials. ${ }^{6,7}$

MSCs act as surveyors in the microenvironment, where as long as there is a local injury MSCs secrete bioactive materials so that they become active and set to repair, regenerate, and regulate the local immune response. The material above is called the secretome, which can be defined as a factor or molecule that is secreted into the extracellular space, where these factors include soluble proteins, nucleic acids, and extracellular vesicles that play a role as immunomodulators. Hence the secretome of MSCs has gained a lot of attention because of its potency in tissue repair and regeneration. ${ }^{5,8}$

MSCs have the potency to repair several kinds of periodontal diseases, aiding in tissue 
repair and regeneration because they have a set of advantages, such as high-quality healing, regenerating damaged tissue without fibrous tissue formation. ${ }^{9}$ Periodontitis is characterised by chronic inflammation caused by bacteria that causes damage to the teeth's supporting structure. Several studies have been able to culture MSCs from periodontal ligament stem cells (PDLSCs) and gingival mesenchymal stem cells (GMSCs) and both of those MSCs have such characteristics similar to MSCs from bone marrow and adipose tissue. Another trait of PDLSCs and GMSCs are the capability to differentiate into specific cells, including cementoblast cells. ${ }^{10-12}$

Even though the secretome role of MSCs is still not clearly understood yet, the emerging concept is that these MSCs contribute to the niche of regulating responses to tissue damage. In this review, we tried to summarise the immunomodulatory function of MSC, factors in the microenvironment that can affect such function, potential applications and challenges as well as its ability to modulate disease progression, such as periodontitis. ${ }^{6}$

\section{Methods}

Several types of research have led to the immunobiology of MSCs, where in vitro research confirms the ability of MSCs to modulate immune responses. MSCs are also involved in pathological and physiological processes depending on tissue damage, cellular homeostasis, inflammatory diseases and ageing. MSCs mobilise towards the site of inflammation by secreting growth factors so that new tissue regeneration is expected. ${ }^{13}$

\section{MSCs activity in the site of inflammation}

MSCs take part in repairing tissue damage. Tissue injuries are thought to be related to the immune system activity or inflammatory cells including CD4 + T cells, CD8 + T cells and B cells, also of the macrophages or neutrophils. Inflammatory mediators including IL- $1 \beta$, TNF- $\alpha$, leukotrienes, chemokines and various free radicals are produced by phagocytic cells each time cellular damage occurs in an organism. Microenvironment can be altered by the action of inflammatory molecules and immune cells, in conjunction with endothelial cells and fibroblasts which produced in the mobilisation and differentiation of MSCs into stromal cells and supplanted the impaired tissues. MSCs generally retrieved from bone marrow or other sources and also be found in tissues, i.e. resident/endogenous MSCs. The mechanism of mobilisation and recruitment of MSCs in cell or tissue damage has not fully endure and transform itself into specific cells are also unclear in the inflammatory site. ${ }^{14,15}$

At the inflammatory site, MSCs also secrete substances i.e IL-1, IFN- $\gamma$, TNF- $\alpha$, which triggered certain growth factors namely vascular endothelial growth factor (VEGF), epidermal growth factor (EGF), platelet-derived growth factor (PDGF), stromal cell-derived factor-1 (SDF-1), insulin growth factor (IGF), fibroblast growth factor (FGF), plus transforming growth factor- $\beta$ (TGF- $\beta$ ). The growth above factors can increase progenitor cells, as well as fibroblast, and endothelial cells development, which can lead to regeneration, which heals the tissues. $^{14,16}$

The reciprocal action between MSCs and inflammatory cells can be complicated. MSCs can either secrete cytokines or inhibit the activity of cytokines. MSCs, secrete TGF- $\beta$ and other factors, thereby increasing the induction of $\mathrm{T}$ cells and macrophages. The paracrine effect of MSCs is essential for maintaining endothelial cell integrity and improving angiogenesis via regulation of endothelial cell proliferation and extracellular matrix production and prevents interactions between leukocyte cells and endothelial cells. Fibroblast cells are essential toward tissue integrity and damage repair as well as secreting metalloproteinase matrix. MSCs activities in homeostatic immune cells including interacting with cytokines, chemokines, and molecules at cell's surface. Previous studies of MSC regulation have focused on interactions between MSCs and B lymphocyte cells, NK cells, and dendritic cells. Whereas recent reviews tend to explore the usage of MSCs to repair tissue damage and regulation of the inflammatory response can improve the functioning of macrophages and T lymphocyte cells. ${ }^{9,17,18}$

Figure 1 MSCs repress neutrophils activation, inhibit activity of Natural Killers, hindering dendritic cells maturation and differentiation of monocyte cells from pro-inflammatory (M1) in favour of anti-inflammatory (M2). They also inhibit the cytotoxic cell cycle of T cells, B lymphocytes, CD8+, increase T helper Th2, Treg, also enhancing the anti-inflammatory CD4 +, and even hamper pro- inflammatory CD4+ T helper Th17 and Th1. ${ }^{19}$

\section{Immunomodulatory properties of MSCs}

MSCs has been intrigued by many researchers as a material for tissue repair. Almost all dental tissues, including periodontium tissue host a population of cells akin to MSC. The oral cavity MSC also has a strong immunomodulatory property. The MSC in oral cavity modulates the adaptive, as well as innate immune response, thus influences the activity of almost all components of the immune system. The reciprocal actions between the oral cavity MSC and the immune system caused by

Journal of Dentomaxillofacial Science (J Dentomaxillofac Sci) December 2021; 6(3): 139-146 | doi: 10.15562/jdmfs.v6i3.989 


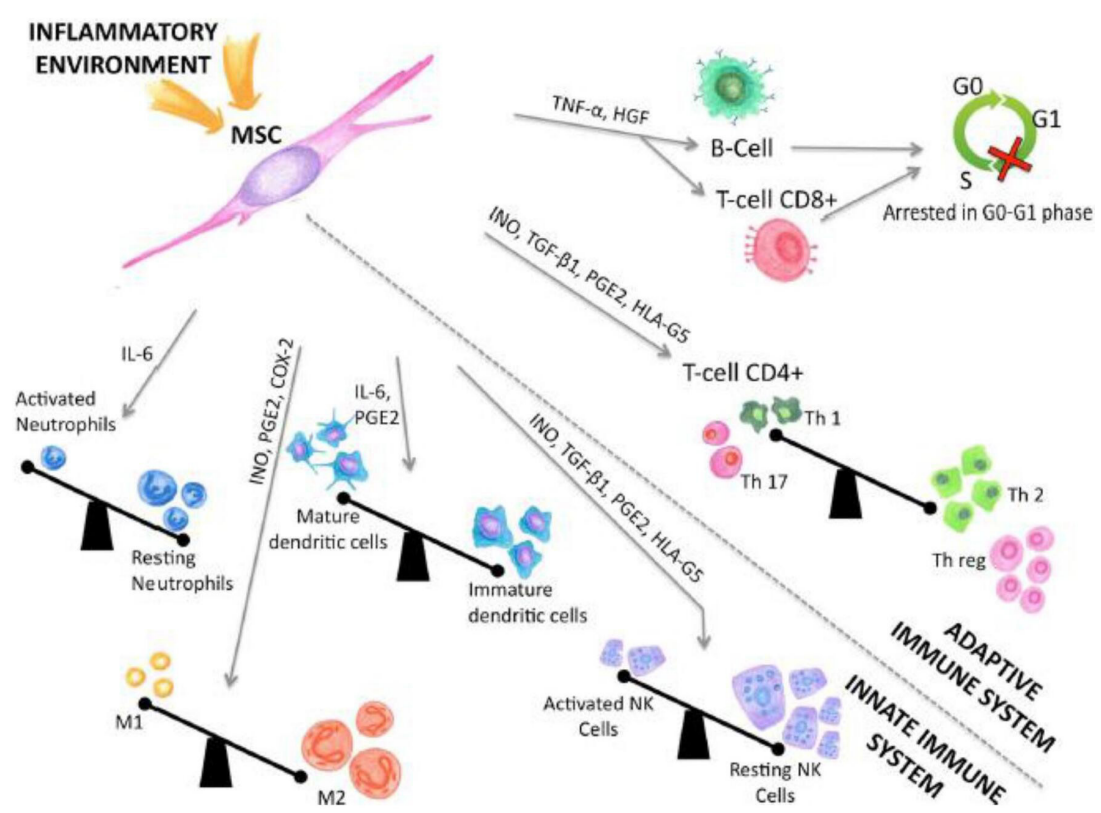

Figure 1 The capability of MSCs in tuning the adaptive as well as the innate immune system in the inflammatory situation.

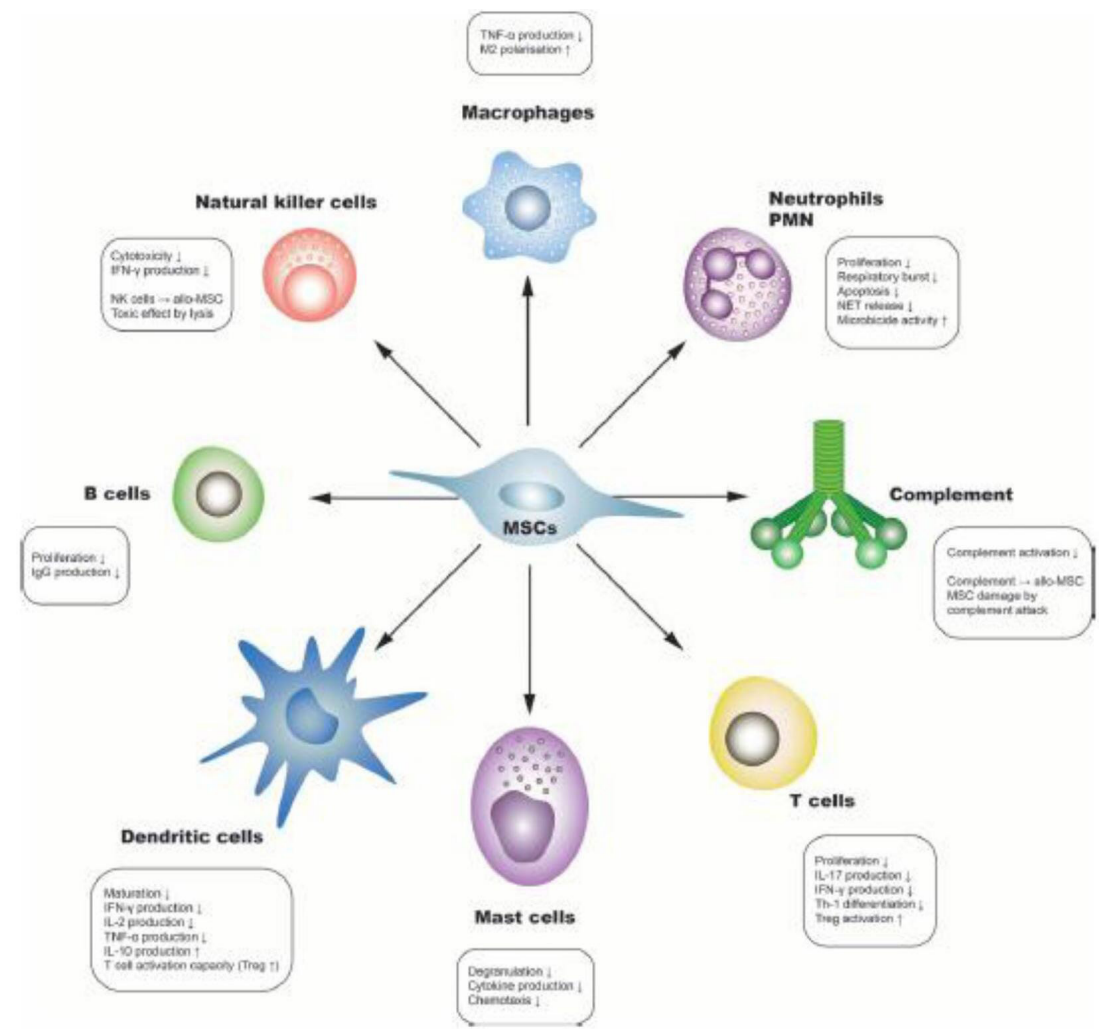

Figure 2 Immunomodulatory MSCs has been known to affect various components in both adaptive and innate immune systems

cavity MSC and the immune system caused by the immunomodulatory activity of the MSC are managed cautiously by cytokines of the immune cells. MSC that taken from inflammatory tissue may show impaired immunomodulatory capacity; this affects cells that play a role in inflammatory diseases, especially periodontitis. The therapeutic effects of MSCs include immunomodulatory capabilities; this consists of the assembly of various growth factors and immunomodulatory proteins. The role of oral MSCs is essential in tissue homeostasis both in healthy and diseased conditions..$^{20,21}$

Oral MSC is situated in the perivascular niche, where the MSCs are still at rest, undifferentiated by the microenvironment. After the injury to the tissue, MSCs cells are recruited into damaged areas and play a role in repair by proliferating and differentiating into several specific cells. Another critical function of MSCs is the modulation of immune and inflammatory responses. MSCs in the perivascular system allows the interaction of the recruitment of MSCs in vivo, as well as the migration and modulation of transvascular area. Inflammations are marked by the presence of chemotaxis from MSCs to the inflammatory site so that the role of MSCs as immunomodulators is able function properly. ${ }^{20}$

The essential substance that takes part in MSCs mediated immunosuppression is indoleamine-2,3dioxygenase (IDO). Tryptophans are catabolized by the intracellular enzyme into kynurenine. Reduced tryptophan results in a reduction in different immune cells. IDO expression is minuscule in MSCs and is exceedingly regulated by interferon (IFN) $-\gamma$ The most important soluble factor mediating the immunomodulatory effect of MSC is interleukin-10, transforming tumour growth factor- $\beta$ (TGF) $-\beta$ and prostaglandin E2 (PGE-2). PGE-2 is an arachidonic metabolic product, whose production is managed by cyclooxygenase 2 and involved in regulation both innate and adaptive immune system by MSCs. A potent immunomodulator namely TGF- $\beta$ is perpetually produced by MSCs and its production can be increased by other anti-inflammatory factors such as IL- 4 and IL-13. IL-10 is an antiinflammatory cytokine, which formed by MSC itself or by immune cells assigned by MSCs. Furthermore, the dissolved factors are also have been known to participate in MSCs mediated immunomodulation, such as human leukocyte antigen (HLA) -G5, galectin and TNF gene $-\alpha .{ }^{22}$

MSCs can influence the proliferation, activation, and function of immune cells. Pre-clinical studies in animal models have shown immunosuppressive effects on adaptive and innate immune systems. Fascinatingly, MSCs usually express major histocompatibility complex I (MHC-I) but do not have MHC-II, CD40, CD80 and CD86 expressions on the surface of the cell, so that MSCs are not recognised by $\mathrm{T}$ cells, causing a reduced immune response in the case of tissue or organ transplant. MSCs are also able to influence the activity and proliferation of immune cells due to a balance between pro- 
inflammatory and anti- inflammatory cells. This is based on research on mouse models of uveitis which treated with MSCs derived from umbilicals which showed significant results in suppressing inflammatory proinflammatory cytokines IL-6, IL-8, tumour nuclear factor- $\alpha$ (TNF- $\alpha$ ) and MIP1 but caused increased expression of IL-antiinflammatory cytokines 10 . MSC can regulate the proliferation, differentiation and activity of most immune cells, such as T cells, B cells, NK cells, dendritic cells (DC), macrophages, via direct or indirect contact with these immune cells. MSCs can produce several bioactive molecules to inhibit inflammation or immune responses. For example, MSC can inhibit the proliferation and activation of $\mathrm{T}$ cells, and induce differentiation and expansion of regulatory $\mathrm{T}$ cells. ${ }^{20,22}$

\section{Factors affecting the immunomodulating function of MSCs}

Several types of research proved that MSC plays the immunosuppressive and anti-inflammatory role. The MSCs "plasticity" in immunomodulatory activities hints that the function of immunomodulators can be enhanced or inhibited by the exposed microenvironment. Microenvironment's inflammatory factors are crucial to affect the immunomodulatory function. For example, interferon- $\gamma$ $(\mathrm{IFN}-\gamma)$ functions as an immunosuppressive in innate and adaptive immune responses. In addition to inflammatory factors, Toll-like receptors (TLRs) and hypoxic environments can alter the immunomodulatory function of MSCs. However, other studies have shown that human bone marrow MSCs are activated with TLR3 or TLR4 ligands to release proinflammation, such as IL-1 $\beta$, IL-6, CXCL10. This effect is mediated by caspase- 8 . Inhibition of caspase- 8 can stimulate an increase in the discharge of anti-inflammatory mediators, such as HGF and TGF- $\beta .{ }^{20,23}$

Recent reports showed that MSCs express tolllike receptors (TLRs), which play an essential role in immunomodulation that clinically established. TLR-3 and TLR-4 ligation (which binds lipopolysaccharides (LPS) and innate self-antigens) block the ability of MSCs to inhibit T-cell responses via $\mathrm{NOTCH}$ signals via down-regulation of Jagged-1 expression in MSCs.

Interestingly TLR-3 was known to mainly mediate the migration of stress response in MSCs. Also, it has been known that MSCs snuffed out cytokine production and T-cell proliferation, which involves mediators IL-10, TGF-b, indoleamine 2, 3-dioxygenase (IDO) and prostaglandin E2 (PGE2) through unexplained mechanisms. The immunosuppressive ability of MSCs is not an innate immune system but rather is caused by pro- inflammatory cytokines: IFN- $\gamma$ is combined with tumour nuclear factor- $\alpha$ ( TNF- $\alpha$,) IL- $1 \alpha$ or IL- $1 \beta$. Because MSCs are responsive to pro-inflammatory cytokines, it is hypothesised that cytokines produced during the immune response prompted the expression of chemokines by MSCs. These chemokines may function to recruit or mobilise the lymphocytes, which is indispensable in MSCs mediated immunosuppression. ${ }^{23,24}$

\section{MSCs as Antimicrobial Agent}

MSCs possess antimicrobial effects through direct and indirect mechanisms mediated by peptide and protein secretion (AMPs). The indirect mechanism by which MSCs regulate the role of the hist system against pathogenic bacteria, in particular, the pro-inflammatory and anti-inflammatory balance of the immune system, or increased phagocytic activity. The direct mechanism by which MSCs secrete antimicrobial materials such as peptides and proteins (AMPs) and also express molecules such as indoleamine 2,3 dioxygenase (IDO) and IL- $17 .{ }^{25}$

Several in vivo researches have shown a favourable effect of MSC therapy in bacterial sepsis cases, indicating the immunomodulatory nature of MSCs due to increased phagocytic activities. Our in vitro studies have shown that human uterine cervical stem cell-conditioned media (hUCSC-CM) exhibit a bactericidal effect on E. coli and S. epidermidis. In vitro research shows that the secretion of hUCSC-CM activates CXCL10, CXCL8, CXCL1, CXCL6, CCL20 and CCL5 which are responsible for this antibacterial effect. This chemokine is proved to possess an antibacterial effect against E. coli and Staphylococcus strains.

MSCs can secrete bioactive molecules such as anti-inflammatory, antimicrobial, angiogenic, chemotaxis, and antiapoptosis. Past studies have shown the ability of MSCs to inhibit the growth of Pseudomonas aeruginosa and Staphylococcus aureus bacteria in vivo. This suggests that MSCs can play a role in gram-negative and positive growth. MSCs can secrete LL-37 peptides as antimicrobials where LL-37's ability to damage bacterial cell walls and LL-37 expression are also stimulated by the presence of inflammatory mediators. Interestingly, LL-37 levels can be increased if stimulated with inflammatory cytokines such as IFN $\gamma$, IL-1 $\beta$, or IL- $12 .{ }^{25,26}$

\section{Results}

Role of MSCs in the Therapy of Periodontal Diseases.

In healthy periodontal tissue, MSCs are in a favourable microenvironment, i.e. self- renewal and 


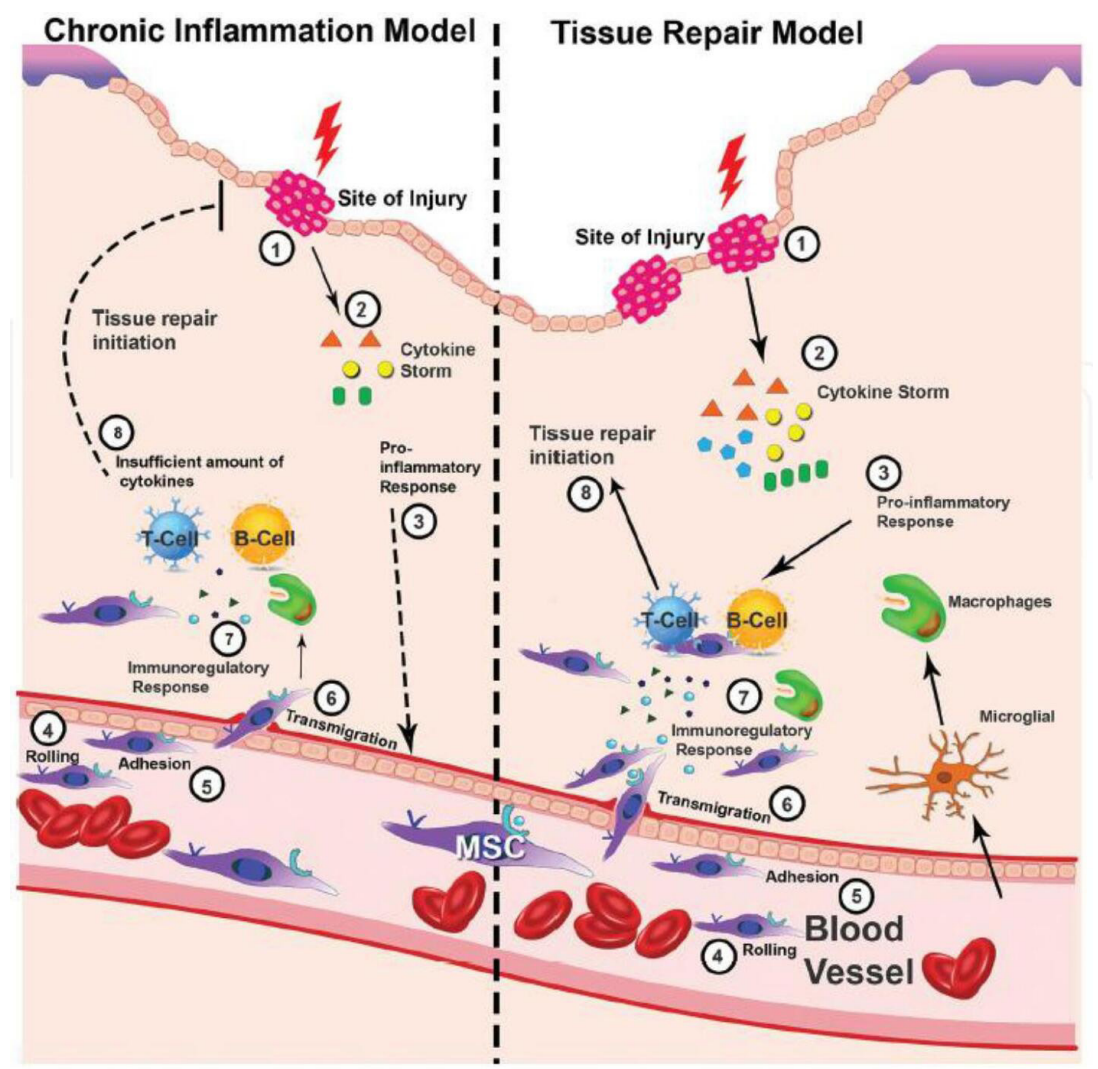

Figure 3 Immunoregulatory action of MSCs in chronic inflammation (left) compared to acute inflammation (right). In the inflammatory site, the activity of immune cells increases so that many cytokines are secreted. Activity and migration from MSCs towards the injury results in MSCs secreting immolators and growth factors depending on cytokine signals (acute inflammation rather than chronic), MSCs begin an immunoregulatory response and repair in the area of injury or are unable to inhibit chronic inflammatory signals that eventually result in cellular fibrosis

periodontal tissue homeostasis. Except, when periodontitis or any other periodontal injuries occur, this favourable microenvironment undergoes many sudden changes involving various types of cells and signalling. Changes in the microenvironment also subsequently change the function of endogenous MSCs. For example, PDLSCs which are obtained from the periodontal microenvironment which currently undergoes inflammation indeed have higher proliferation capability, but its immunomodulatory potential, osteogenic, as well as the adipogenic effect, is lower compared to the healthy PDLSCs. Besides, PDLSCs taken from inflammation exhibited an exceptional proliferative and migration abilities albeit with lesser osteogenesis when compared to healthy PDLSCs from the same patient. And furthermore, in vivo studies show that PDLSCs from inflamed source possess a lower bone regeneration ability following the transplantation in rat model subcutaneously. ${ }^{27,28}$
Observations from several types of research describe PDLSc from experimental animals as having immunomodulatory effects on the administration of xenogenic and allogeneic peripheral blood mononuclear cells caused by inhibition of cell division rather than by apoptosis. Compared with healthy cells, PDLSc cells show significantly reduced T-cell proliferation. In co-cultures, peripheral blood of stimulated mononuclear cells showed substantially less induction of regulatory T-cells, CD4 +, CD25 + and IL-10 secretion in inflammatory areas compared with healthy PDLSc cells. Furthermore, suppression of Th17 differentiation and IL-17 production by inflammatory PDLSc is significantly lower than those in healthy cells. ${ }^{29,30}$

Previous researches have shown a combination of periodontal ligament stem cells (PDLSCs) from mice with platelet-rich fibrin (PRF) transplanted in alveolar bone defects of mandibular first molar mandibular mice caused by drilling. After days 12 and 24 after transplantation, the $\mu$-CT examination revealed that the formation of periodontal ligaments showed new cementum and new alveolar bone on day 24 compared to control and treatment using PRF alone. ${ }^{34}$ The results of research on rat alveolar bone regeneration showed that the average area of trabeculae increased on day 14 and continued to improve on day 28. The increased area of trabeculae showed the formation and mineralisation of new alveolar bone after the administration of MSCs composite seeded with demineralized dentin matrix scaffold. ${ }^{31}$

Periodontitis therapy with surgery and antiinflammatory medications, or a combination of both, has been a conventional approach to the treatment of periodontitis. However, this therapy is effective for controlling periodontitis, but not enough for periodontal tissue regeneration. The administration of exogenous MSCs is expected to be able to activate endogenous MSCs through paracrine signalling to encourage and enhance the host's immune system to be active and trigger the process of periodontal tissue regeneration. ${ }^{32}$

Current researches demonstrate that paracrine effects of secretome MSCs are essential in repair and regeneration after stem cell therapy. Secretome cells consist of a group of extracellular proteins, growth factors, cytokines and chemokines and several molecules that can affect the microenvironment and directly modulate endogenous MSCs. The latest paradigm in which MSCs function as medicinal signalling cells in vivo is not only increasing and differentiating into such unipotent cells or progenitors but also secreting bioactive materials that modulate things at the inflammatory area. ${ }^{4,33}$

According to research results back in the year 2000, MSCs have differentiated properties into 
progenitor cells such as cardiomyocytes, hepatocytes, osteoblasts, adipocytes, chondrocytes, and some cells that are directed to differentiate for in vitro therapy. Research has led to in vivo were using experimental animals for the application of MSCs towards replacement tissue directly. Here it is assumed the ability of MSCs to change the microenvironment through paracrine and autocrine signalling in the host so that it is expected to have a repair and regeneration effect on the tissue. ${ }^{34}$

The potency of MSCs as a therapy in cases of inflammation happens through three mechanisms. The first is by "homing", in which stem cells that administered systemically migrate to the inflammatory area. Signal homing such as stromalderivedfactor 1 (SDF-1) is a chemokine that will bind to CXCR4 will attract stem cells for migration, proliferation and differentiation into bone-forming cells that followed by the distinction into several kinds of cells, which can function locally to heal and replace damaged tissues. The third mechanism is by secreting bioactive factors, which potentially affect the microenvironment in the inflammatory area. ${ }^{13,35}$

Recent research shows the paracrine effect of secretory MSCs is an essential factor in regeneration and repair following stem cell therapy. The role of secretory MSCs for tissue healing is by inducing inflammatory mediators, i.e. interleukin-1 (IL1), IL-6, prostaglandin E2 (PGE2) in vitro and decreasing the initial inflammatory reaction in vivo by modulating pro-inflammatory cytokines i.e. inhibiting TNF- $\alpha$ IFN- $\gamma$ ), IL-1 and increase antiinflammatory secretion namely IL-10 and IL-4. Interleukin-10 that mediate MSCs to regulate T-cell activation and differentiation. ${ }^{26,36}$

Periodontitis is marked by chronic inflammation and several causative factors which are difficult to control. Therefore the combination of immunomodulatory and regenerative abilities of MSCs makes it a promising new candidate for long-term treatment of inflammatory diseases including periodontitis. MSCs modulate inflammation, the host's immune system, and repair damaged tissue, so MSCs have the potency to become the new cellular therapies for chronic inflammation therapy. ${ }^{6,37}$

\section{Discussion}

Secretomes and conditioned-media from MSCs as a new therapeutic strategy

Periodontal disease is caused by oral bacteria and systemic immune responses, the role of oral pathogenic bacteria especially porphyromonas gingivalis, can cause traumatic injury and irritation of the epithelium and mucosa, and play a role in the development of systemic disease. The most common systemic diseases are obesity, hypertension and diabetes mellitus so that they can affect the healing process. ${ }^{38}$

Secretomes are described as an array of factors or secreted molecules that went into the extracellular space. The factors include free nucleic acids, lipids, extracellular vesicles, and several soluble proteins. Extracellular vesicles can be partitioned into several apoptotic bodies, exosomes, and microparticles. In specific tissue areas, Secretomes will change the response to physiological and pathological conditions. $^{39}$

Cell-based therapies that utilized MSC derived from secretome in regenerative medicine yields major advantages over stem cell-based applications: the administration of secretome is safe to use, especially related to cell transplantation and proliferation, especially in terms of rejection ability, tumorigenicity, embolism formation and transmission of infection; secretions originating from MSC are also evaluated for safety, dosage and potential, if analogized with pharmaceutical ingredients; storage, can be carried out in the absence of cryopreservative agents which are potentially toxic for long periods of time without losing the ability of these ingredients; using a secretome sourced from MSC, such as conditioned-medium (CM) is more cost-effective and feasible for clinical applications because it avoids invasive procedures; the time and cost of maintaining cultured stem cells can be subtracted and the secretome that is always readily available can be used for the treatment of acute conditions such as cerebral ischemia, and myocardial infarction; finally, biological products retrieved for therapeutic applications can be modified to various cells of interest. ${ }^{40,41}$

Clinical studies in humans are very limited to the usage of secretome products from MSC. The administration of the Adipose-derived stem cellcontaminated media (ADSC-CM) following the procedure of $\mathrm{CO}^{2}$ laser in human skin, there was an increase of wound healing rate and reduced the effects of hyperpigmentation and erythema. Moreover, intradermal injection of ADSC-CM into the scalp of patients affected by alopecia using a split-scalp study design significantly promotes hair growth in patients of both genders. Similarly, retrospective studies and observations of hair loss patterns of women treated with ADSC-CM showed efficacy after 12 weeks, significantly escalating fullness and density of the hair without any adverse reactions. BMMSC-CMs had been used to assist alveolar bone regeneration safely. A number of clinical applications of MSC-derived exosomes have been reported with prior study shows that increasing the dose of exosome derived from MSC 
in patients with stage IV host-versus-graft disease that is resistant to treatment, affects the skin and gastrointestinal tract, is well tolerated and shows significant improvement, and remains stable for five months- long. ${ }^{26,42}$

\section{Conclusion}

MSCs can act as immunomodulators in periodontal disease by secreting secretomes that alter the microenvironment of inflammatory periodontal tissue so that they can activate exogenous and endogenous MSCs and bioactive molecules that directly increase the potential of MSCs and create a favourable niche for periodontal tissue regeneration.

\section{Acknowledgment}

The authors would also like to thank Islamic development Bank grants.

\section{Conflict of Interest}

The authors report no conflict of interest.

\section{References}

1. Sohni A, Verfaillie CM. Mesenchymal stem cells migration homing and tracking. Stem Cells Int 2013;2013: 14- 16.

2. Katsuda T, Kosaka N, Takeshita F, et al. The therapeutic potential of mesenchymal stem cell-derived extracellular vesicles. Proteomic 2013;00: 1- 17.

3. Caplan AI. Mesenchymal stem cells: time to change the name!. Stem Cells Transl Med 2017;6: 1445- 1451.

4. Somoza RA, Correa D, Caplan AI. Roles for mesenchymal stem cells as medicinal signaling cells. Nat Protoc 2015;6: 2015.

5. Sheard J. From fibroblasts to medicinal signalling cells: a paradigm shift in translational stem cell research. InDepth Focus 2018;2: 42- 22.

6. Sargent A, Miller RH. MSC therapeutics in chronic inflammation. Curr Stem Cell Reports 2016;2: 168- 173.

7. Caplan AI, Dennis JE. Mesenchymal stem cells as trophic mediators. J Cell Biochem 2006;98: 1076- 1084.

8. Vizoso FJ, Eiro N, Cid S, et al. Mesenchymal stem cell secretome: toward cell- free therapeutic strategies in regenerative medicine. Int J Mol Sci 2017;1852:1- 24.

9. Sanz, Antonio R, Carrion, et al. Mesenchymal stem cells from the oral cavity and their potential value in tissue engineering. Periodontol 2000 2015;67: 251- 267.

10. Panduwawala $\mathrm{CP}, \mathrm{Zhan} \mathrm{X}$, Dissanayaka WL, et al. In vivo periodontal tissue regeneration by periodontal ligament stem cells and endothelial cells in three-dimensional cell sheet constructs. J Periodontal Res 2017;52: 408- 418.

11. Grawish ME. Gingival-derived mesenchymal stem cells: an endless resource for regenerative dentistry. World J Stem Cells 2018;10: 116- 118.

12. Nugraha AP, Narmada IB, Ernawati DS, et al. Gingival mesenchymal stem cells from Wistar Rat's Gingiva (Rattus Novergicus) - Isolation and characterization (in vitro study). J Int Dent Med Res 2018;11: 694- 699.

13. Herrero C, Pérez-Simón JA. Immunomodulatory effect of mesenchymal stem cells. Brazilian J Med Biol Res 2010;43: 425- 430.

14. Ma S, Xie N, Li W, et al. Immunobiology of mesenchymal stem cells. Cell Death Differ 2014;21: 216- 225.
15. Stoddart MJ, Bara J, Alini M. Cells and secretome towards endogenous cell re-activation for cartilage repair. Advanced Drug Delivery Reviews 2015;84:135- 145.

16. Ayala-Cuellar AP, Kang JH, Jeung EB, et al. Roles of mesenchymal stem cells in tissue regeneration and immunomodulation. Biomol Ther 2019;27: 25- 33.

17. Abdulmajid, Niyaz A. Paracrine effects of mesenchymal stem cells on dental tissues-in vitro and in vivo. Dissertation 2016:1- 124.

18. Haojiang L, Shi S, Haitao F, et al. Immunomodulatory functions of mesenchymal stem cells in tissue engineering. Stem Cells Int 2019;2019: 967- 1206.

19. Pierro M, Thébaud B. MSCS in Scenarios of infection and inflammation: focus on neonatal diseases. Curr Stem Cell Reports 2016;2: 158- 167.

20. Andrukhov O, Behm C, Blufstein A, et al. Immunomodulatory properties of dental-derived mesenchymal stem cells. In: Periodontology. London: IntechOpen; 2018. p. 1- 31.

21. Wada N, Gronthos S, Bartold PM. Immunomodulatory effects of stem cells. Periodontol 2000 2013;63: 198- 216.

22. Zhaoa Q, Renb H, Hana Z. Mesenchymal stem cells: immunomodulatory capability and clinical potential in immune diseases. J Cell Immunother 2016;2: 3- 20.

23. Qu G, Xie X, Li X, et al. Immunomodulatory function of mesenchymal stem cells: regulation and application. J Cell Immunother 2018;4: 1- 3.

24. Kode JA, Mukherjee S, Joglekar MV, et al. Mesenchymal stem cells: immunobiology and role in immunomodulation and tissue regeneration. Cytotherapy 2009;11: 377- 391 .

25. Sutton MT, Fletcher D, Ghosh SK, et al. Antimicrobial Properties of Mesenchymal Stem Cells: Therapeutic Potential for Cystic Fibrosis Infection, and Treatment. Stem Cells Int 2016;2016: 1- 12.

26. Alcayaga-Miranda F, Cuenca J, Khoury M. Antimicrobial activity of mesenchymal stem cells: Current status and new perspectives of antimicrobial peptide-based therapies. Front Immunol. 2017;8: 1- 15.

27. An Y, Liu W, Xue P, et al. Increased autophagy is required to protect periodontal ligament stem cells from apoptosis in inflammatory microenvironment. J Clin Periodontol 2016;43: 618- 625

28. Lei M, Li K, Li B, et al. Mesenchymal stem cell characteristics of dental pulp and periodontal ligament stem cells after in vivo transplantation. Biomaterials 2014;35: 6332- 6343.

29. Liu D, Xu J, Liu O, et al. Mesenchymal stem cells derived from inflamed periodontal ligaments exhibit impaired immunomodulation. 2012: 1- 9 .

30. Wada N, Gronthos S, Bartold PM. Immunomodulatory effects of stem cells. Periodontol 2000 2013;63: 198- 216.

31. Duan X, Lin Z, Lin X, et al. Study of platelet-rich fibrin combined with rat periodontal ligament stem cells in periodontal tissue regeneration. J Cell Mol Med 2018;22: 1047- 1055.

32. Sari DS, Maduratna E, Ferdiansyah, et al. Osteogenic Differentiation and Biocompatibility of Bovine Teeth Scaffold with Rat Adipose-derived Mesenchymal Stem Cells. Eur J Dent, 2019; 13:206- 212.

33. Linero I, Chaparro O. Paracrine effect of mesenchymal stem cells derived from human adipose tissue in bone regeneration. PLoS One 2014;9: 1- 12.

34. Holan V, Hermankova B, Bohacova P, et al. Distinct Immunoregulatory Mechanisms in Mesenchymal Stem Cells: Role of the Cytokine Environment. Stem Cell Rev Rep 2016;12: 654- 663 .

35. Hill ABT, Bressan F, Murphy BD, et al. Application of Mesenchymal stem cell technology in Bovine Spesies. Stem Cells Research and Therapy 2019; 10:1- 13. 
36. Perez RA, Seo SJ, Won JE, et al. Therapeutically relevant aspects in bone repair and regeneration. Mater Today 2015;18: 573- 589.

37. Lin L, Du L. The role of secreted factors in stem cells-mediated immune regulation. Cell Immunol 2018;326: 24- 32.

38. Aji NR, Herawati D. Association between periodontal disease and prostate cancer. J Dentomaxillofac Sci 2019;4: 1-

39. Gao F, Chiu SM, Motan DAL, et al. Mesenchymal stem cells and immunomodulation : current status and future prospects. Cell Death Dis 2016;7: e2062.

40. Park Se-Ra, Kim Jae-Wan, Jun Hee-Sook, et al. Stem cell secretome and its effect on cellular mechanisms relevant to wound healing. Mol Ther 2018;26: 606- 617.

41. Vizoso FJ, Eiro N, Cid S, et al. Mesenchymal stem cell secretome: toward cell-free therapeutic strategies in regenerative medicine. Int J Mol Sci 2017;18.
42. Madrigal M, Rao KS, Riordan NH. A review of therapeutic effects of mesenchymal stem cell secretions and induction of secretory modification by different culture methods. J Transl Med 2014;12: 1- 14.

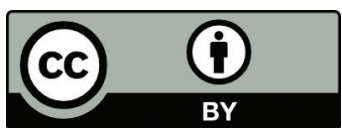

This work is licensed under a Creative Commons Attribution 